\title{
CONTROL CHANNEL BASED MAC-LAYER CONFIGURATION, ROUTING AND SITUATION AWARENESS FOR COGNITIVE RADIO NETWORKS ${ }^{\ddagger}$
}

\author{
Srinivasan Krishnamurthy, Mansi Thoppian, S. Venkatesan, and Ravi Prakash \\ School of Engineering and Computer Science \\ ksrini@ student.utdallas.edu, \{mansi, venky, ravip\}@utdallas.edu \\ The University of Texas at Dallas, Richardson, TX-75083
}

\begin{abstract}
In a Cognitive Radio (CR) network, MAClayer configuration involves determining a common set of channels to facilitate communication among participating nodes. Further, the availability of multiple channels and frequent channel switches add to the complexity of route selection. Knowledge of the global network topology can be used to solve the above-described problems. In this paper, we propose a distributed algorithm for gathering global network topology information for a CR network. We outline approaches that utilize the gathered topology information for MAC-layer configuration and efficient routing of packets. In addition, situation awareness is achieved by sharing the physical location information among the nodes in the network. The proposed algorithm determines the global network topology in $O\left(N^{2}\right)$ timeslots, where $N$ is the maximum number of nodes deployed. With 80 available channels for communication, the algorithm terminates within 0.8 second.
\end{abstract}

\section{INTRODUCTION}

Cognitive Radio (CR) technology [11] offers a new mechanism for flexible usage of radio spectrum. Even though the number of available frequency bands is decreasing [6], considerable portions of the frequency spectrum at a given place at any given time are not fully utilized [7], [16]. The CR approach allows secondary users to operate in such under-utilized licensed frequency bands in an intelligent way without constraining the privileges of licensed (primary) users [16]. A CRenabled secondary user (referred to as a node in the rest of paper) is capable of periodically scanning and identifying available channels in the frequency spectrum. Channel $\mathrm{c}$ is said to be available if a secondary user can transmit and receive messages on c for a reasonable amount of time without interference to/from the primary users. Defense and relief operations could greatly benefit from such a communication infrastructure. As the set of available channels could change over time and from one region to another [7], it is necessary to use dynamic channel assignment schemes for communication among the $\mathrm{CR}$ nodes.

$\ddagger$ Supported in part by NSF CAREER grant no. CCR-0093411 and by STTR Grants from NIST and ONR.
In a CR network, each node scans the radio spectrum and determines the set of available channels independently. This gives rise to the following MAC-layer issues:

(i) How do nodes identify their neighbors and communicate with them?

(ii) How do nodes decide on the set of channel(s) that can be used for communication across the entire network?

In this paper we address the above-mentioned issues and propose a distributed MAC-layer configuration scheme. We also consider the problem of routing in a CR network. As opposed to traditional networks, a CR network has the ability to use multiple communication channels within the same network. Since transmissions on different channels do not interfere with each other, using multiple channels in parallel increases the network throughput. In a CR network, the number of available channels is not fixed and the available channels can be anywhere in the entire spectrum. This is in contrast to the existing use of multiple channels, where the number of channels and their positions in the frequency spectrum is fixed. When the network has the ability to utilize multiple channels, traditional routing metrics such as number of hops, congestion, energy used etc., are not sufficient to make correct routing decisions. A CR network introduces some new route metrics for a link. These include:

1) Number of channel switches along a path from the source to destination nodes.

2) Frequency of channel switches on a link. In a CR network, there is no guarantee that a channel will be available for use for the entire communication duration between two nodes. A channel can become unavailable due to the return of primary user or due to congestion. If a channel becomes unavailable on a path, then that link will be considered broken and all the packets for that link will be dropped. Therefore, it is important to consider how often a channel becomes unavailable on a link while computing the path between source and destination nodes. 


\section{A. Motivation}

Wireless communication among nodes in a CRnetwork can be facilitated by (i) a channel common to all the nodes to exchange control information, (ii) a set of channels at each node that it can use to exchange data with its neighbors, (iii) the ability to determine and use an alternate global control channel if the original control channel is jammed, and (iv) a mechanism to determine efficient routes based on the channels available on each link in the network. Each node in the network is initially assumed to be aware of a globally common control channel and the set of channels available to itself for data communication. The set of channels that the node then uses for exchanging data with its neighbors can either be a set common to all nodes in the network (referred to as the global channel set, $\mathcal{G}$, in this paper) or a set that is common to its k-hop neighborhood (referred to as the $k$-local channel set, $\mathcal{L}_{k}$, in this paper). Note that $\mathcal{G}=\mathcal{L}_{D}$, where $D$ is the diameter of the network. The incentives for finding the set $\mathcal{G}$ are the following:

- At the site of a natural disaster or in a military operation, multiple groups of nodes that handle specific (and most likely separate) functions could be deployed. Control information among the nodes in each group should propagate without any interference from other groups in the vicinity. Thus, each group needs to choose a unique channel for communication among its members. Note that a few nodes in each group could act as gateways for intergroup communication.

- Switching from one channel to another results in non-zero delay. This channel switching delay depends on the relative positions of the two channels on the radio spectrum. For example, tuning delays could be of the order of $1 \mathrm{~ms}$ for a $10 \mathrm{MHz}$ step in the frequency range $20 \mathrm{MHz}-3 \mathrm{GHz}$ [5]. By using a single globally common channel for communication, such switching overheads can be avoided.

- For mobile ad hoc networks (MANETs), where the network topology changes dynamically, a globally common channel for communication among all the nodes is preferable ([12], [15]) and numerous researchers in the MANET community have assumed this model.

- If the globally common control channel becomes unavailable, one of the channels from $\mathcal{G}$ can be selected as the new control channel.

The reasons for finding the set $\mathcal{L}_{k}$ are the following:

- $\mathcal{G}$ may be an empty set. However, that does not preclude data communication among the nodes.
Note that a non-empty $\mathcal{L}_{1}$ at each node in the network is sufficient for effective communication.

- Good utilization of available channels. Using locally common channels enables parallel communication among nodes in close proximity if they employ different frequencies to avoid interference.

Determining $\mathcal{G}$, and $\mathcal{L}_{k}$ for any arbitrary $k$ can be done effectively by providing the nodes with the global network topology. In addition, by exchanging GPS positional data among nodes, each node can be made aware of the physical location of the other nodes in the network. This information is particularly useful in hostile and chaotic environments such as urban warfare, where correctly identifying a friend from a foe is of paramount importance.

\section{B. Our contribution}

Let $N$ be the total number of possible nodes and $M$ be the total number of possible channels (in addition to a control channel) the nodes can operate on. In this paper, we propose a MAC-layer configuration protocol that enables the nodes to dynamically discover the global network topology in a distributed manner, provided all nodes are aware of $N$ and $M$. We also present routing strategies based on the number of channel switches along a path and frequency of channel switches over a link. The proposed MAC-layer configuration requires $O\left(N^{2}\right)$ timeslots. For as many as 40 nodes $^{1}$ and 80 channels [13] and the timeslot duration being $0.5 \mathrm{msec}$, the configuration protocol terminates in 0.8 second. As a byproduct of our algorithm, situation awareness (SA) data is also propagated.

Key contributions of this paper are:

- The proposed MAC-layer configuration algorithm enables nodes to dynamically discover the global network topology and physical location of each node in the network. The physical location is useful for SA.

- The configuration algorithm identifies the set of channels that are common to all the nodes of the network.

- The proposed routing strategy enables the use of routing metrics such as the number of channel switches along a path and frequency of channel switches over a link for finding "best" routes and thus addresses constraints unique to $\mathrm{CR}$ networks.

The rest of this paper is organized as follows. Section II presents the system model and assumptions. The proposed MAC-layer auto-configuration protocol and routing strategies are described in detail in Section III.

\footnotetext{
${ }^{1}$ In an army, a platoon consists of around forty soldiers ([4], [2]).
} 
We discuss alternatives to the proposed protocol for certain scenarios in Section IV and Section V concludes the paper.

\section{SYSTEM MODEL}

Throughout this paper, we consider a mobile multihop wireless network formed by a group of CR-enabled nodes.

\section{A. Node characteristics}

We assume that $N$ is fixed and known a priori to all the nodes in the network. This assumption is justified as the applications under consideration are military and relief operations, where the maximum number of soldiers in a platoon or the maximum number of firemen assigned for a relief task is known a priori. The identities of nodes are unique and are pre-assigned from the range $[1 \ldots N]$. The nodes are assumed to be GPS-equipped [9] to enable physical location awareness and time synchronization among nodes. This is a reasonable assumption considering that it has become a standard procedure for personnel involved in military and disaster relief operations to carry devices that have GPS capability ([8]). Each node is equipped with one configurable IEEE 802.11a [3] wireless card in addition to a transceiver capable of operating over the frequencies available for a CR network. The IEEE $802.11 a$ cards provide the nodes with a globally common control channel that the nodes are aware of as soon as they are turned on. The cards are configurable in the sense that they will use a contentionfree slotted scheme for communication instead of the usual contention-based scheme employed by such cards. Please refer to Section II-C for the details of the scheme.

\section{B. Medium characteristics}

The communication medium is assumed to be lossfree. In a lossy environment, the proposed algorithm is assumed to run on top of a reliable communication mechanism. Let $\mathcal{A}_{\text {univ }}=\left\{C_{1}, C_{2} \ldots C_{M}\right\}$ be the universal set of channels that are potentially available to a node in the CR network. Thus, $M=\left|\mathcal{A}_{\text {univ }}\right|$. We assume that $\mathcal{A}_{\text {univ }}$ is known a priori to all the nodes in the network. For example, $\mathcal{A}_{\text {univ }}$ can be composed of a set of 80 channels in the $3-5 \mathrm{GHz}$ frequency range. A unique identity is assigned to every channel and the identities are known in advance to all nodes. In addition to the channels in $\mathcal{A}_{\text {univ }}$, a separate control channel is used for exchanging control messages.

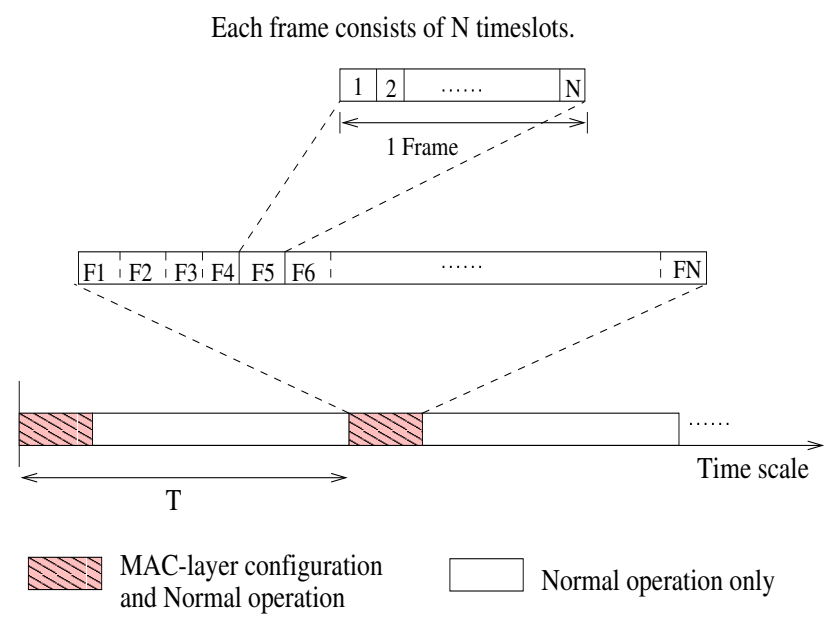

Fig. 1. Operation cycle of the network

\section{Network operation}

At any given instant of time, nodes in the CR network are operating in either of the following two modes: (i) control channel based MAC-layer configuration and normal operation, or (ii) normal operation only. The nodes repeat MAC-layer configuration protocol every $T$ time units, as illustrated in Figure 1. The time instant at which each execution of the configuration protocol is invoked is assumed to be known a priori to all the nodes in the network. Such an assumption is feasible because of the presence of GPS capability in the nodes. The value of $T$ could be hard-wired into the nodes or set just before deployment of the nodes. During the MAClayer configuration operation, nodes use customized IEEE 802.11a cards for exchanging control messages. Employing IEEE 802.11a cards ensures that every node is tuned to the same control channel during MAClayer configuration operation. These cards are specially configured to use TDMA-based slotted mechanism instead of the standard CSMA/CA protocol. During MAClayer configuration, nodes learn the global network topology as well as the global channel set, $\mathcal{G}$. Node behavior during normal mode of operation is comparable to that of a node in other multi-hop wireless networks, such as MANET [17] or mesh networks [10]. We require that the nodes invoke the MAC-layer configuration operation every $T$ time units to maintain accuracy despite changes in network topology, changes in channel availability set maintained by individual nodes, and/or node movements. When a CR node is turned on, it remains silent until the first execution of the MAC-layer configuration protocol.

\section{MAC-LAYER CONFIGURATION AND ROUTING}

During MAC-layer configuration, time is split into intervals referred to as frames as shown in Figure 1. 
Each frame is further divided into $N$ timeslots, each of equal length. The slot assignment for a node, say $i$, is done in advance according to its identity. For simplicity, we assume that the identity of node $i$ is $i$. Node $i$ is allowed to transmit during the $i^{\text {th }}$ slot in each frame (see Figure 1 ) and all other nodes are in receive mode (during the $i^{t h}$ slot). This ensures that every node in the network gets one chance to transmit without collisions during each frame.

\section{A. Data structures}

The following data structures are maintained at every node $i$ :

$U I D^{i}$ An $N$-element array of node identities

$\mathcal{A}^{i} \quad$ An array of $N$ elements where the $j^{t h}$ element, $\mathcal{A}_{j}^{i}$ corresponds to the channel availability set of node $j$ as known to node $i$

$A D J^{i}$ An array of $N$ elements where the $j^{t h}$ element, $A D J_{j}^{i}$ corresponds to the adjacency list of node $j$ as known to node $i$

$G P S^{i}$ An array of $N$ elements where the $j^{t h}$ element, $G P S_{j}^{i}$ corresponds to the GPS location of node $j$ as known to node $i$ and is a 5 -tuple of the form < latitude, latitude hemisphere, longitude, longitude hemisphere, height $>^{2}$

$\mathcal{G} \quad$ Global channel set

$\mathcal{A}_{i}^{i}$ and $G P S_{i}^{i}$ of every node $i$ are populated as a bootstrap process prior to the initiation of the configuration protocol. Initially, $U I D_{i}^{i}=i, U I D_{j}^{i}=-1 \forall j \neq i$, $\mathcal{A}_{j}^{i}=\emptyset \forall j \neq i$ and $G P S_{j}^{i}=\emptyset \forall j \neq i$.

\section{B. MAC-layer configuration}

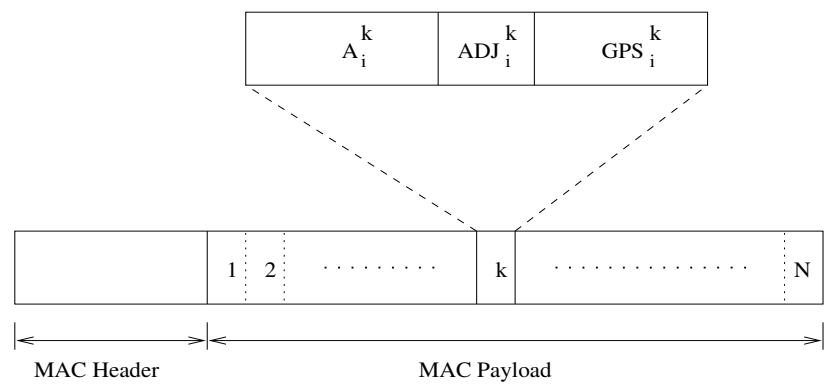

Fig. 2. Packet transmitted by node i

Every node $i$ transmits a packet of the form illustrated in Figure 2 during $i^{\text {th }}$ timeslot of each frame. The

\footnotetext{
${ }^{2}$ Latitude, $\{$ ddmm.mmmm $\}$, where $\mathrm{d}$ and $\mathrm{m}$ represent degrees and minutes respectively; latitude hemisphere (N/S); Longitude, \{dddmm.mmmm\}; longitude hemisphere (E/W) and height, [-9999.9, 99999.9] [1].
}

transmitted payload is divided into $N$ segments. Segment $k$ corresponds to what node $i$ knows about node $k$. At the time of transmission, if node $i$ is unaware of node $k$, then the corresponding segment consists of a sequence of zero bits. This is referred to as $S E G 0$. During the remaining time slots of a frame, node $i$ is in the receive mode. At the end of each frame, node $i$ updates $U I D_{i}, \mathcal{A}_{i}$, $A D J_{i}$ and $G P S_{i}$ by using each packet received during that frame. For a packet received from node $j$, node $i$ updates its data structures as follows:

begin

for $k=1$ to $N$

If $k^{t h}$ received segment $\neq S E G 0$ and $U I D_{k}^{i}=-1$, then

$$
\begin{aligned}
& U I D_{k}^{i} \leftarrow k \\
& \mathcal{A}_{k}^{i} \leftarrow \mathcal{A}_{k}^{j} \\
& A D J_{k}^{i} \leftarrow A D J_{k}^{j} \\
& G P S_{k}^{i} \leftarrow G P S_{k}^{j}
\end{aligned}
$$

end

Thus, at the end of $N$ frames, each node $i$ knows the availability sets, GPS locations and adjacency information of all the nodes in the network. Node $i$ then sets the global channel set $\mathcal{G}$ to $\cap_{\forall j} \quad U I D_{j}^{i} \neq-1 \mathcal{A}_{j}^{i}$ and constructs the global network topology based on received data. The data structures are reset to their initial values every $T$ time units before the initiation of the configuration algorithm. This is done to ensure that the data structures are properly updated and retain only the information obtained from the latest execution of the configuration protocol.

\section{Complexity analysis}

The channel availability set and adjacency list information of each node requires a total of $M$ bits and $N$ bits respectively. The number of bits required for the GPS positional data of each node can be calculated as follows. The latitude information is sent as the binary equivalent of the integral number obtained as $(d d+m m . m m m m / 60) \times 10^{4}$ (see Section III-A for explanation). Since the maximum possible value is 90000000 , the binary representation of latitude will require 27 bits. Similarly, the longitude information is sent as the binary equivalent of the integral number obtained as $(d d d+m m . m m m m / 60) \times 10^{4}$. Here, the maximum possible value is 180000000 and will require 28 bits in binary representation. The latitude and longitude hemispheres information are each sent as one bit. The height information is sent as the binary equivalent of a 6 -digit integer with a maximum value of 999999 . This will require 21 bits including the sign bit. Thus, the GPS location information of a single node requires a total of 78 bits $(27+1+28+1+21)$. For $N=40$, 
$M=80$, during each timeslot, a total of 7920 bits $\left[\mid\left(G P S_{i} \mid+M+N\right) \cdot N=(78+80+40) \cdot 40\right]$ need to be transmitted. At a transmission rate of $54 \mathrm{Mbps}$ [3], this information can be transmitted in about $0.15 \mathrm{msec}$. To account for the preamble required to establish message bit synchronization, and guard bands for synchronization error and propagation time [3], we will assume a timeslot duration of $0.5 \mathrm{msec}$. The MAC-layer configuration operation requires $N$ frames ( $N^{2}$ timeslots) for completion. Thus, the running time of the MAC-layer configuration operation is $1600 \mathrm{X} 0.5 \mathrm{msec}=0.8$ seconds.

\section{Routing}

Based on the global topology information collected, each node can compute the "best" routes to all nodes in the network. In addition to using the number of hops as metric for route selection, we propose the use of other routing metrics mentioned earlier in the paper such as the number of channel switches along a route and/or frequency of channel switches over a link. Based on the these two metrics, we propose two new routing strategies.

1) Routing based on minimum latency: Consider a node $i$ along the path from source to destination. If the incoming and outgoing links of node $i$ use different channels, then there is a channel switch at node $i$. A channel switch incurs some overhead and increases the latency and end-to-end delay. The time required for a channel switch depends on the relative positions of the two channels on the radio spectrum. Switching takes negligible time if the two channels are very close to each other; otherwise, the switching time can be significant. Note that the shortest path in terms of the number of hops may not always yield the path with minimum number of channel switches. On the other hand, a path with minimum number of channel switches may be very long for practical purposes. Therefore, the route computation has to be based on a trade-off between the length of the path and the number of channel switches. Using the global topology information collected during MAC-layer configuration process, a source node can compute the route to destination as the path that minimizes the overall latency. Overall latency of a packet transmission on a path can be computed as follows. Suppose a path, $P$, from source node $S R C$ to destination node $D S T$, consists of $d$ hops and $s$ channel switches. Let $t_{h o p}$ and $t_{s w}$ be the 1hop propagation delay and channel switch delay respectively. Then the overall latency, $T_{l a t}$ of $P$ is given by

$$
T_{l a t}=d \times t_{h o p}+s \times t_{s w}
$$

The source node will select the path with minimum $T_{\text {lat }}$ and forward the packet to the next hop node along the selected path ${ }^{3}$. Since every node knows the global topology, the next hop node can perform path computation from $S R C$ to $D S T$ independently. The fact that all nodes have identical data (at the end of each MAC layer configuration period) ensures that there is no inconsistency and there will be no loops when packets are routed using the results of the routing algorithm.

2) Routing based on frequency of channel switches over links: To account for the possibility of a link becoming unusable, every node maintains a weighted average of the duration for which each channel is available along each outgoing link. Based on the weighted averages maintained, a node can compute the probability of a channel being available on a link. Every time a node has a packet to transmit, it selects the channel with the highest probability ${ }^{3}$. Since every node makes a local decision about the channel to use for transmission, this strategy might result in a path with high number of channel switches. The appropriate strategy would be to use this approach in conjunction with the routing scheme described earlier.

\section{E. Situation Awareness}

The commonly used definition for Situation Awareness (SA) is "the perception of the elements in the environment within a volume of time and space, the comprehension of their meaning and the projection of their status in the near future"[14]. SA is important for effective decision making and performance in warfare and emergency rescue and relief operations, where CR networks are likely to be deployed. During the MAClayer configuration protocol described in this paper, besides exchanging network topology information, nodes also propagate GPS positional data. Thus, every node in the network is aware of the physical locations of other nodes in the network and can be said to possess a limited amount of Situation Awareness. This information could be extremely useful in military operations in chaotic environments where distinguishing a friend from a foe is a major challenge. Recent friendly-fire incidents involving U.S troops and its allies in Afghanistan and

\footnotetext{
${ }^{3}$ The transmitting node informs the receiving node on what channel the packet will be transmitted by using the control channel.
} 
Iraq that have resulted in tragic loss of lives underscore the importance of SA. Note that the periodic re-running of the MAC-layer configuration protocol ensures that the physical location information is fairly up-to-date.

\section{DISCUSSION}

Although the $5 \mathrm{GHz}$ band employed by $802.11 a$ is not as heavily used as the $2.4 \mathrm{GHz}$ band used by the more popular $802.11 b$ and $802.11 \mathrm{~g}$ standards, there might be situations where the $802.11 a$ cards may not be usable or may even be deliberately jammed. In such scenarios, the auto-configuration protocol described in [13] can be used. The protocol described in that work assumes that the nodes are equipped with only one transceiver, which is effectively the case when the $802.11 a$ band is jammed. At any given instant in time, the nodes can be engaged either in auto-configuration operation or normal operation and not both. The auto-configuration protocol determines $\mathcal{G}$ in $O(N \cdot(M+D))$ timeslots, where $D$ is the diameter of the network. The auto-configuration protocol consists of two phases. The second phase ( $D N$ timeslots) is almost identical to the protocol described in this paper. The main difference is that instead of all nodes transmitting on a single common control channel in their respective timeslots, each node transmits on the channel that is common to itself and its neighbors i.e. a channel in $\mathcal{L}_{1}$. The first phase ( $M N$ timeslots) involves determining $\mathcal{L}_{1}$ at every node and propagating it to its neighbors. The details of the auto-configuration protocol appear in [13].

\section{CONCLUSion}

In this paper, we addressed the MAC-layer configuration and routing problem in a CR network and presented a distributed algorithm for obtaining global network topology and physical location of nodes in the network. We assumed that nodes have no prior knowledge of their neighborhood and are aware of a globally common control channel. Using our algorithm, all nodes in the network determine the global network topology in $O\left(N^{2}\right)$ timeslots. For reasonable network deployment scenarios, the time taken is less than a second. Nodes could determine the global channel set using the network topology information. In scenarios where this set is empty, the proposed algorithm enables every node to compute the set of channels that is common to itself and all other nodes within its $k$-hop neighborhood. Such locally common channel sets facilitate establishing communication infrastructure among subsets of nodes connected through gateways. We also proposed routing strategies using metrics that are particularly relevant to CR networks. A byproduct of our algorithm is that SA information is readily available to all the nodes in the network.

\section{ACKNOWLEDGMENTS}

We would like to thank Jeff Barton of Rockwell Collins Inc. for providing valuable information about frequency spectrum usage and also answering several questions about cognitive radio technology. We would also like to thank Mansoor Mohsin and Maulin Patel for their helpful suggestions during initial brain-storming sessions.

\section{REFERENCES}

[1] GPS 35 LP Technical Specification, Webpage http://www.garmin.com/manuals/GPS35LPSeries_Technical Specification.pdf.

[2] MSN Encarta Dictionary, Webpage http://encarta.msn.com/dictionary_l861738652/platoon.html.

[3] Supplement to Part 11 standard for Wireless LAN Medium Access Control (MAC) and Physical (PHY) Layer Specifications - High speed physical layer in the $5 \mathrm{GHz}$ band, ANSI/IEEE Std 802.11a-1999.

[4] Wikipedia - The Free Encyclopedia. Article on Platoon, Webpage - http://en.wikipedia.org/wiki/Platoon.

[5] TCI 8067 Spectrum processor data specification, Webpage http://www.tcibr.com/PDFs/8067webs.pdf, 2000.

[6] FCC, Spectrum Policy Task Force Report, ET Docket No. 02135, November 2002.

[7] FCC, Spectrum Policy Task Force Report, ET Docket No. 02155, November 2002.

[8] Frost and Sullivan's North American GPS equipment markets, Report A601-22, 2003.

[9] A. S. Brown. Embedding GPS receivers in software defined radio. In Proceedings of Cognitive Radios, March 2004.

[10] Ian F. Akyildiz, Xudong Wang and Weilin Wang. Wireless mesh networks: a survey. In Elsevier Computer Networks, pages 143, January 2005.

[11] J. Mitola. Cognitive radio: An integrated agent architecture for software-defined radio, 2000.

[12] Jinyang Li, Charles Blake, Douglas S.J. De Couto, Hu Imm Lee and Robert Morris. Capacity of ad Hoc wireless networks. In Proceedings of the $7^{\text {th }}$ annual international conference on Mobile computing and networking, pages 61-69, July 2001.

[13] S. Kuppa, M. Thoppian, S. Krishnamurthy, S. Venkatesan, R. Chandrasekaran, N. Mittal, and R. Prakash. Time-efficient layer-2 auto-configuration for cognitive radios. Technical Report UTDCS-20-05, The Univ. of Texas at Dallas, Richardson, TX, May 2005.

[14] M. Endsley. Design and evaluation for situation awareness enhancement. In Proceedings of the Human Factors Society 32nd Annual Meeting, pages 97-101, October 1988.

[15] Matthias Grossglauser and David N. C. Tse. Mobility increases the capacity of ad hoc wireless networks. In IEEE/ACM Transactions on Networking, pages 477-486, August 2002.

[16] R. W. Broderson et al. Corvus: A cognitive radio approach for usage of virtual unlicensed spectrum, Webpage http://bwrc.eecs.berkeley.edu/Research/MCMA/CR_White_paper finall.pdf.

[17] S. Corson and J. Macker. Mobile Ad Hoc Networking (MANET): Routing Protocol Performance Issues and Evaluation Considerations. In RFC 2501, January 1999. 